\title{
Hábitos alimentarios y estado nutricional en pacientes diabéticos con retinopatía que acuden a una Clínica Oftalmológica Privada de la ciudad de Asunción
}

\author{
*Eliana Meza Miranda ${ }^{1}$, Beatriz Elizabeth Nuñez ${ }^{2}$, María Serafini ${ }^{3}$, Antonella Vacchetta ${ }^{3}$ \\ ${ }^{1}$ Universidad Nacional de Asunción, Centro Multidisciplinario de Investigaciones Tecnológicas. Paraguay \\ ${ }^{2}$ Universidad Americana. Paraguay \\ ${ }^{3}$ Universidad de la Integración de las Américas. Facultad de la Salud. Paraguay
}

\begin{abstract}
Cómo referenciar este artículo/
How to reference this article:
\end{abstract}

\begin{abstract}
Meza Miranda E, Nuñez BE, Serafini M, Vacchetta A. Hábitos alimentarios y estado nutricional en pacientes diabéticos con retinopatía que acuden a una Clínica Oftalmológica Privada de la ciudad de Asunción. Mem. Inst. Investig. Cienc. Salud. 2019; 17(2): 64-70
\end{abstract}

\section{RE S U M E N}

La retinopatía diabética (RD) es una complicación microvascular crónica, específica de la diabetes. Es una causa importante de pérdida visual en adultos, con consecuencias médicas, sociales y financieras significativas. El objetivo fue evaluar los hábitos alimentarios y estado nutricional en diabéticos con retinopatía que acuden a la Clínica Vista 10 de la ciudad de Asunción entre setiembre y noviembre del año 2018. Estudio descriptivo, observacional de corte transverso que incluyó 50 pacientes, Siendo la mayor parte de ellos del sexo femenino (65\%), la edad media fue de $62,8 \pm 11,3$ años, el $66 \%(n=33)$ estaba casado con nivel educativo primario $(n=20 ; 40 \%)$ y vivía en Gran Asunción $(n=46 ; 92 \%)$. Respecto a la frecuencia de consumo de alimentos, se encontró que casi la totalidad de la población realizaba el desayuno (96\%). El 70\% consumía lácteos descremados menos de la cantidad recomendada. Solo un $20 \%$ consumía frutas todos los días. Un poco más de la mitad (52\%) consumía verduras todos los días. El $70 \%$ consumía pescado por debajo de las recomendaciones establecidas, y el $78 \%$ consumía legumbres menos de la cantidad recomendada. Más de la mitad (58\%) consumía alimentos integrales por debajo de las recomendaciones. El 54\% consumía comida casera todos los días y el $56 \%$ realizaba la cena todos los días. Más de la mitad de los participantes presentó sobrepeso u obesidad. Los hábitos de la población son deficientes, en relación al consumo de frutas, lácteos según las recomendaciones de las Guías Alimentarias del Paraguay.

Palabras clave: retinopatía, hábitos alimentarios, diabetes.

\section{Food habits and nutritional status in diabetic patients with retinopathy that attend a private Ophthalmological Clinic of the city of Asunción}

\section{A B S T R A C T}

\section{ABSTRACT}

Diabetic retinopathy (RD) is a chronic microvascular complication specific to diabetes. It is an important cause of visual loss in adults, with significant medical, social and financial consequences. The objective was to evaluate the dietary habits and nutritional status in diabetic patients with retinopathy who attend the Clínica Vista 10 in the city of Asunción between September and November of 2018. This was a descriptive, observational crosssectional study The majority of the population studied was female ( $n=33,65 \%)$, the mean age was $62.8 \pm 11.3$ years, $66 \%(n=33)$ was married and the highest percentage was presented primary education level $(n=20,40 \%)$. According to the classification of the place of origin, most lived in Gran Asunción ( $n=46,92 \%)$. Regarding the frequency of food consumption, it was found that almost the entire population made breakfast $(96 \%), 70 \%$ 
consumed low-fat dairy products less than the recommended amount. Only $20 \%$ consumed fruits every day. A little more than half (52\%) consumed vegetables every day, $70 \%$ consumed fish below the established recommendations and $78 \%$ consumed legumes less than the recommended amount. More than half (58\%) consumed whole food below the recommendations, 54\% consumed home-cooked food every day and $56 \%$ made dinner every day. More than half of the population was overweight or obese. The habits of the population are deficient, in relation to the consumption of fruits, dairy products according to the recommendations of the Food Guides of Paraguay.

Keywords: retinopathy, dietary habits, diabetes.

\section{INTRODUCCIÓN}

Varios factores ambientales (externos) y genéticos (internos) están involucrados en el desarrollo de la retinopatía diabética (RD). La detección adecuada y la detección temprana de tales factores mejorarán la prevención y el tratamiento de esta enfermedad cegadora ${ }^{(1)}$.

La prevalencia de la diabetes está aumentando debido a la mayor sobrevida y al cambio en el estilo de vida de la población, llegando incluso a más del $10 \%$ en algunos países. Después de 20 años, el $90 \%$ de los casos de diabetes tipo 1 y el $60 \%$ del tipo 2 tendrían alguna forma de retinopatía y de ellas, el 5\% requerirá de tratamiento para evitar una ceguera irreversible. La RD es la tercera causa de ceguera irreversible en el mundo, pero la primera en personas de edad productiva (16 a 64 años) en países en vías de desarrollo, generando grandes pérdidas económicas ${ }^{(2)}$.

La RD es la complicación más común de la diabetes y sigue siendo una causa importante de ceguera prevenible. Se producen cambios anatómicos y funcionales en varias células de la retina, incluidas las células endoteliales de la retina, las neuronas y el epitelio pigmentario de la retina antes de los síntomas clínicos de la enfermedad. Los cambios tempranos incluyen la aparición de microaneurismas, la adhesión de leucocitos, la apoptosis de las células vasculares (endoteliales y pericitos) y las células neuronales ${ }^{(3)}$. Los cambios progresan hasta involucrar la ruptura de las barreras retinianas internas y externas de la sangre que causan edema macular diabético, la causa más importante de pérdida de visión en la RD. La degeneración capilar y el desarrollo de los capilares acelulares conducen al deterioro de la perfusión retiniana y, posteriormente, a la hipoxia y la neovascularización retiniana, el sello distintivo de la retinopatía diabética proliferativa (RDP) ${ }^{(3)}$.

Por otro lado, la Asociación Americana de Diabetes y la Asociación Europea para el Estudio de la Diabetes establecen que la terapia nutricional juega un papel importante en la prevención de complicaciones relacionadas con el control y control metabólico de la enfermedad ${ }^{(4)}$.

En términos de nutrición se ha visto que existe un mayor riesgo de progresión de la DR en aquellos sujetos con mayor ingesta de ácidos grasos y una asociación de menor riesgo entre aquellos con mayor ingesta de fibra dietética. Por otro lado, un estudio con un diseño transversal observó un efecto protector de la ingesta de ácidos grasos monoinsaturados (MUFA) y ácidos grasos poliinsaturados (PUFA) para la RD en pacientes bien controlados $^{(4)}$.

Como hemos visto, la nutrición juega un papel importante en la progresión de la RD en esta población, por lo tanto, el objetivo de este trabajo fue el de evaluar los hábitos alimentarios y estado nutricional en diabéticos con retinopatía que acuden a la Clínica Vista 10 de la ciudad de Asunción.

\section{MATERIALES Y MÉTODOS}

El diseño del presente estudio es de tipo observacional descriptivo transversal. Se evaluaron 50 pacientes adultos diabéticos con retinopatía que acudieron a la Clínica Vista 10 de Asunción entre setiembre y noviembre del año 2018 y se excluyeron a pacientes mayores de 65 años y mujeres embarazadas o en periodo de lactancia. Fueron estudiadas variables sociodemográficas, dietéticas (hábitos alimentarios) y antropométricas (estado nutricional). Se accedió a la Clínica Vista 10 mediante una nota de permiso dirigida a los jefes de dicha clínica, posteriormente se reclutó a la población accesible que cumplía con los criterios de inclusión en el consultorio a los cuales mediante una nota de consentimiento informado se les informó sobre la importancia de la participación en el estudio, y posteriormente se realizó el relevamiento de los datos.

Se utilizó una ficha elaborada por las investigadoras en donde se recogieron datos personales, sociodemográficos y dietéticos de los pacientes. 
La presente investigación se realizó según las normas del Departamento de Salud y Servicios Humanos (DHHS) de los Estados Unidos, de forma a garantizar que la investigación sea éticamente aceptable.

Se respetaron los tres principios básicos de; el principio de respeto a las personas, el de beneficencia y el de justicia.

\section{RESULTADOS}

\section{Características sociodemográficas}

La mayor parte de la población estudiada fue del sexo femenino $(n=33 ; 65 \%)$, la edad media fue de $62,8 \pm 11,3$ años, el $66 \%(n=33)$ estaba casado y el mayor porcentaje presentaba nivel educativo primario $(n=20 ; 40 \%)$. Según la clasificación del lugar de procedencia la mayor parte vivía en Gran Asunción $(n=46 ; 92 \%)$. La ocupación laboral predominante fue la de trabajadores no calificados $(n=20 ; 40 \%)$.

Los resultados respecto a las características sociodemográficas se observan en la Tabla 1.

Tabla 1: Datos sociodemográficos

\begin{tabular}{llrr}
\hline Datos sociodemográficos & $\mathbf{n}$ & $\mathbf{\%}$ \\
\hline Sexo & Masculino & 17 & 34 \\
& Femenino & 33 & 65 \\
\hline Estado civil & Soltero/a & 9 & 18 \\
& Casado/a o unión libre & 33 & 66 \\
& Separado/a o divorciado/a & 1 & 2 \\
& Viudo/a & 7 & 14 \\
\hline Nivel & Primaria & 20 & 40 \\
& Secundaria & 17 & 34 \\
& Terciaria & 13 & 26 \\
& Profesionales científicos e intelectuales. & 13 & 26 \\
\hline Ocupación & Trabajadores de los servicios y & 15 & 30 \\
& vendedores de comercios y mercados. & 1 & 2 \\
& Agricultores y trabajadores calificados & 20 & 40 \\
& agropecuarios y pesqueros. & 1 & 2 \\
\hline Total & Trabajadores no calificados. & 46 & 92 \\
& Fuerzas armadas. & 4 & 8 \\
\hline Procedencia & Gran Asunción & $\mathbf{5 0}$ & $\mathbf{1 0 0}$ \\
\hline & Interior del país & & \\
& & &
\end{tabular}

\section{Grado de severidad de retinopatía diabética}

En cuanto al grado de severidad de retinopatía diabética se encontró que la mayor parte de la población estaba dentro de la clasificación de "Sin retinopatía aparente"en un 66\% $(n=33)$, seguido por "Retinopatía diabética proliferativa" en un $18 \%(n=9)$. Ver Tabla 2. 
Tabla 2: Grado de retinopatía de la población

\begin{tabular}{lrr}
\hline Grado de severidad & $\mathbf{n}$ & \% \\
\hline Sin retinopatía aparente & 33 & 66 \\
Retinopatía no proliferativa leve & 6 & 12 \\
Retinopatía no proliferativa moderada & 1 & 2 \\
Retinopatía no proliferativa severa & 1 & 2 \\
Retinopatía diabética proliferativa & 9 & 18 \\
TOTAL & $\mathbf{5 0}$ & $\mathbf{1 0 0}$ \\
\hline
\end{tabular}

\section{Frecuencia de consumo de alimentos}

Respecto a la frecuencia de consumo de alimentos, se encontró que casi la totalidad de la población realizaba el desayuno (96\%). El 70\% consumía lácteos descremados menos de la cantidad recomendada. Solo un $20 \%$ consumía frutas todos los días. Un poco más de la mitad (52\%) consumía verduras todos los días. El 70\% consumía pescado por debajo de las recomendaciones establecidas y $78 \%$ consumía menos legumbres de la cantidad recomendada. Más de la mitad (58\%) consumía alimentos integrales por debajo de las recomendaciones. El 54\% consumía comida casera todos los días mientras que el $56 \%$ realizaba la cena todos los días (ver Tabla 3).

Tabla 3: Frecuencia de consumo de alimentos (1)

\begin{tabular}{llll}
\hline & $\begin{array}{l}\text { No } \\
\text { consume/nunca }\end{array}$ & $\begin{array}{l}\text { Ocasional/menos } \\
\text { de lo } \\
\text { recomendado }\end{array}$ & $\begin{array}{l}\text { Todos los días/ } \\
\text { porciones ideales } \\
\text { al día }\end{array}$ \\
\hline Consumo de desayuno & $0(0 \%)$ & $2(4 \%)$ & $48(96 \%)$ \\
Lácteos descremados & $7(14 \%)$ & $35(70 \%)$ & $8(16 \%)$ \\
Frutas & $0(0 \%)$ & $40(80 \%)$ & $10(20 \%)$ \\
Verduras & $0(0 \%)$ & $24(48 \%)$ & $26(52 \%)$ \\
Pescados & $12(24 \%)$ & $35(70 \%)$ & $3(6 \%)$ \\
Legumbres & $7(14 \%)$ & $39(78 \%)$ & $4(8 \%)$ \\
Alimentos integrales & $3(6 \%)$ & $29(58 \%)$ & $18(36 \%)$ \\
Comida casera & $1(2 \%)$ & $22(44 \%)$ & $27(54 \%)$ \\
Consumo de cena & $10(20 \%)$ & $12(24)$ & $28(56 \%)$ \\
\hline
\end{tabular}

Por otro lado, $76 \%$ de la población refirió no consumir bebidas azucaradas, no obstante $20 \%$ refirió consumirlas hasta dos porciones por día. En cuanto al alcohol, $66 \%$ no lo consumía, no obstante, un 32\% lo consumía hasta dos porciones por día. El $76 \%$ de la población consumía frituras hasta dos porciones por día y $60 \%$ no consumía sal. La mitad de la población refirió consumir comida rápida hasta dos porciones por día y más de la mitad (58\%) bocadillos dulces hasta dos porciones por día (ver Tabla 4). 
Tabla 4: Frecuencia de consumo de alimentos (2)

\begin{tabular}{llll}
\hline & $\begin{array}{l}\text { No } \\
\text { consume/nunca }\end{array}$ & $\begin{array}{l}\text { Menos de 1 o } \\
\text { hasta 2 } \\
\text { porciones/día }\end{array}$ & $\begin{array}{l}\text { Siempre/3 o } \\
\text { más } \\
\text { porciones/día }\end{array}$ \\
\hline Bebidas azucaradas & $38(76 \%)$ & $10(20 \%)$ & $2(4 \%)$ \\
Alcohol & $33(66 \%)$ & $16(32 \%)$ & $1(2 \%)$ \\
Frituras & $11(22 \%)$ & $38(76 \%)$ & $1(2 \%)$ \\
Sal & $30(60 \%)$ & $14(28 \%)$ & $6(12 \%)$ \\
Comida rápida & $24(48 \%)$ & $25(50 \%)$ & $1(2 \%)$ \\
Bocadillos dulces & $15(30 \%)$ & $29(58 \%)$ & $6(12 \%)$ \\
\hline
\end{tabular}

\section{Estado nutricional}

En cuanto al estado nutricional hemos encontrado que solo un $28 \%$ de la población se encontraba con peso adecuado al momento de la evaluación. 34\% presentó sobrepeso, $20 \%$ obesidad grado I, $10 \%$ obesidad grado II y $8 \%$ obesidad grado III (ver Tabla 5 ).

Tabla 5: Estado nutricional de la población

\begin{tabular}{lrr}
\hline Estado nutricional & $\mathbf{n}$ & \% \\
\hline Bajo peso & 0 & 0 \\
Adecuado & 14 & 28 \\
Sobrepeso & 17 & 34 \\
Obesidad grado I & 10 & 20 \\
Obesidad grado II & 5 & 10 \\
Obesidad grado III & 4 & 8 \\
TOTAL & $\mathbf{5 0}$ & $\mathbf{1 0 0}$ \\
\hline
\end{tabular}

\section{DISCUSIÓN}

Según reportes de la OMS, el número de diabéticos en el mundo aumentará a 366 millones de personas en el año 2030. Se presentará así una epidemia de Diabetes Mellitus, la que ya está en curso, lo que implicaría también una epidemia de Retinopatía Diabética con una gran cantidad de personas ciegas o discapacitadas visuales en etapa laboral activa.

Uno de los pilares fundamentales para la prevención y tratamiento de la DM es la alimentación adecuada por lo que en el presente trabajo se valoraron los hábitos alimentarios y estado nutricional en diabéticos con retinopatía.

En primer lugar, hemos caracterizado a la población sociodemográficamente y hemos encontrado que la edad promedio fue de 62,8 años, resultado similar encontrado por Prado et al., quienes encontraron que la edad promedio en una población con retinopatía diabética fue de 65,5 años $^{(5)}$. Por otro lado, este estudio reveló que la mayor parte fue del sexo femenino en una proporción del $65 \%$, resultado que coincide con el de Prado et al. , quienes hallaron en su estudio una proporción del $61 \%$ del sexo femenino ${ }^{(5)}$.

En cuanto al grado de severidad de retinopatía diabética se encontró que la mayor parte de la población se encontró dentro de la clasificación de "Sin retinopatía aparente" en un $66 \%(n=33)$, resultado ampliamente superado por el de McCarty et al., quienes encontraron en su estudio, una prevalencia de pacientes sin retinopatía aparente de $89 \%$ $(n=65)$. Además, este trabajo evidenció un $12 \%(n=6)$ de retinopatía no proliferativa leve, porcentaje superado por lo encontrado por McCarty quien encontró un 28,6\% de pacientes con este diagnóstico ${ }^{(6)}$. El otro grado de severidad encontrado por este estudio fue el de "Retinopatía diabética proliferativa" en una proporción de $18 \%(n=9)$, cifra que supera a lo hallado por McCarty et al. quienes encontraron un $12,5 \%(n=1)$ de sujetos con este grado de severidad de retinopatía ${ }^{(6)}$. 
En cuanto a la frecuencia de consumo de alimentos, hemos encontrado que casi la totalidad de la población realiza el desayuno. El desayuno se considera una comida muy importante en el control de la glicemia y prevención de la DM y no realizarlo se considera un factor de riesgo para el desarrollo de esta enfermedad ${ }^{(7)}$. Casi las tres cuartas partes consume lácteos descremados por debajo de las recomendaciones. Aunque no está claro el mecanismo, varios estudios sugieren que el consumo de lácteos mejora el metabolismo de la glucosa ya que tienen un efecto insulinotrópico, además, aparentemente, los productos lácteos con bajo contenido de grasa tienen una influencia positiva en la prevención y tratamiento de la DM por lo cual se debe aumentar su consumo ${ }^{(8)}$. Solo un $20 \%$ consume frutas todos los días y en contrapartida un poco más de la mitad consume verduras todos los días. En pacientes con diabetes, es muy importante el consumo de frutas y verduras, no solo por el aporte de fibra a través de éstos, sino porque su consumo supone una reducción de la incidencia de enfermedades cardiovasculares en ellos ${ }^{(9)}$. El $70 \%$ consumía pescado por debajo de las recomendaciones. Según varios estudios, el consumo de pescados marinos en diabéticos tiene efectos protectores contra la disfunción de células pancreáticas a través de varios mecanismos mejorando así la resistencia a la insulina ${ }^{(10)}$. Setenta y ocho por ciento consumía legumbres menos de la cantidad recomendada. Varios estudios demostraron que la incorporación de legumbres como parte de una dieta con un índice glicémico bajo, mejora tanto el control de la glicemia como la reducción de la puntuación de riesgo de enfermedad cardiovascular en la $\mathrm{DM}^{(11)}$. Más de la mitad (58\%) consumía alimentos integrales por debajo de las recomendaciones. En personas diabéticas el consumo de alimentos integrales es necesario ya que sus componentes tienen acciones muy importantes, por ejemplo, la ingesta de zinc mejora sutilmente la secreción de insulina, el ácido ferúlico defiende contra los estresores de las células beta, el magnesio mejora la acción de la insulina y el resultado es que los niveles de glucosa permanecen normales ${ }^{(12)}$. Más de la mitad consumía comida casera todos los días. Es de suma importancia que los diabéticos consuman alimentos mínimamente procesados, ya que éstos son más saciantes y menos hiperglicémicos que los alimentos ultraprocesados que ofrece la industria alimenticia ${ }^{(13)}$. El $56 \%$ realizaba la cena todos los días. Las personas que padecen de diabetes deben realizar todas las comidas del día para mantener sus niveles de glicemia en niveles normales, por lo tanto, la cena es una de las comidas que debe realizar, no obstante, lo que sugieren los estudios es que se haga una combinación de un desayuno rico en energía con una cena de bajo consumo energético lo cual disminuiría la hiperglucemia diaria global en estos pacientes ${ }^{(14)}$.

Por otro lado, este estudio reveló que un no despreciable $20 \%$ de la población consumía hasta dos porciones de bebidas azucaradas y un $58 \%$ bocadillos dulces hasta dos porciones por día. En este sentido, el consumo de azúcar, específicamente las bebidas endulzadas con azúcar, ha sido ampliamente responsabilizado del aumento mundial de la diabetes tipo 2, además, la Organización Panamericana de la Salud limita a los azúcares agregados a un consumo no mayor o igual al $10 \%$ del total de calorías diarias por lo que se debe controlar su ingesta ${ }^{(15)}$. Respecto al consumo de alcohol, hemos encontrado que $32 \%$ lo consumía hasta dos porciones por día. El consumo moderado de alcohol tiene relación con la aparición de enfermedades cardiovasculares, neuropatías y complicaciones en retinopatías diabéticas así como problemas neuroendócrinos, gastrointestinales e interacciones con la medicación del paciente diabético, por lo tanto, se deben ofrecer estrategias de evaluación e intervención para disminuir su consumo ${ }^{(16)}$. Tres cuartas partes de la población consume frituras hasta dos porciones por día. El consumo de alimentos fritos se relaciona con un aumento del riesgo de insuficiencia cardiaca por lo que estos pacientes deben evitar la ingesta de este tipo de alimentos ${ }^{(17)}$. La mitad de los pacientes refirió consumir comida rápida hasta dos porciones por día. Los hallazgos de un estudio indicaron que el consumo de comida rápida induce una mayor carga de trabajo cardíaco en general en personas con DM 2 en comparación con personas sanas ${ }^{(18)}$.

En cuanto al estado nutricional, $72 \%$ de los pacientes presentó exceso de peso ya sea por sobrepeso u obesidad, resultado sobrepasado por el encontrado por Firuozi et al., quienes encontraron un $86,5 \%$ de diabéticos con exceso de peso ${ }^{(19)}$. La coexistencia del exceso de peso y la diabetes abre un nuevo concepto en el manejo de esta enfermedad que es la "diabesity" (diabetes + obesidad), la misma supone una epidemia moderna que enlaza varios mecanismos fisiopatológicos alrededor de la resistencia a la insulina y la hiperglicemia, por lo tanto, en vista a su alta prevalencia y sus efectos negativos, es de suma importancia establecer estrategias de intervención tanto farmacológicas como 
dietéticas en estos pacientes y llevar un estricto control en ellos de manera a lograr una buena adherencia al tratamiento ${ }^{(20)}$.

Como fortaleza de este trabajo cabe resaltar que es el primero en realizarse en el país con énfasis en pacientes con retinopatía diabética el cual puede utilizarse como antecedente para la realización de otros trabajos en el área.

Como limitación del trabajo se resalta la escasez de estudios sobre hábitos alimentarios en pacientes con retinopatía diabética tanto a nivel nacional como internacional que permitan adquirir un conocimiento más extendido sobre el tema.

Para finalizar, en continuidad de este trabajo se podrían analizar las mismas variables pero dentro de un diseño prospectivo que permita determinar si influye o no en la progresión o aparición de la retinopatía diabética.

\section{REFERENCIAS BIBLIOGRÁFICAS}

1. Petrovski G, Kaarniranta K, I DP, Szent-gy A. Oxidative Stress, Epigenetics, Environment, and Epidemiology of Diabetic Retinopathy. J Diabetes Res (Internet). 2017; Disponible en: https://www.ncbi.nlm.nih.gov/pmc/articles/ PMC5340930/pdf/JDR2017-6419357.pdf

2. Asociación Panamericana de Oftalmología PAAO Programa Visión 2020/IAPB Latinoamérica Consejo Internacional de Oftalmología ICO. Guías Clínicas para el manejo de la patología ocular del diabético (nternet). 2017. Disponible en: http://www.icoph.org/downloads/ICOGuideli nesDiabeticEyeCare2017-Spanish.pdf

3. Al-shabrawey $M$, Zhang $W$, Mcdonald D. Diabetic Retinopathy: Mechanism, Diagnosis, Prevention, and Treatment. Biomed Res Int (Internet). 2015; Disponible en:

https://www.ncbi.nlm.nih.gov/pmc/articles/ PMC4383145/pdf/BMRI2015-854593.pdf

4. Granado-casas M, Ram A, Mart M, Real J, Alonso N, Valldeperas $X$, et al. Type 1 Diabetic Subjects with Diabetic Retinopathy Show an Unfavorable Pattern of Fat Intake. Nutrients. 2018;10(9):1-10.

5. Carrasco P. E CN. Manejo nutricional de la diabetes tipo 2 en el adulto. Índice glicémico de los alimentos. Rev La ALAD. 2017;7(3):111-9.

6. Mccarty DJ, Fu CL, Franzco CAH, Franzco HRT, Mph CAM. Five-year incidence of diabetic retinopathy in the Melbourne Visual Impairment Project. Clin Exp Ophthalmol. 2003;31(5):397-402.

7. Bi H, Gan Y, Yang C, Chen Y, Tong X, Lu Z. Breakfast skipping and the risk of type 2 diabetes: A meta-analysis of observational studies. Public Health Nutr. 2015;18(16):3013-9.

8. Bjørnshave A, Hermansen K. Effects of dairy protein and fat on the metabolic syndrome and type 2 diabetes. Rev Diabet Stud. 2014;11(2):153-66.

9. Tanaka S, Yoshimura Y, Kamada C, Tanaka S, Horikawa C, Okumura R, et al. Intakes of dietary fiber, vegetables, and fruits and incidence of cardiovascular disease in Japanese patientswith type 2 diabetes. Diabetes Care. 2013;36(12):3916-22.
10. Yuzuru Iizukaa, Hyounju Kima, Takuya Izawaa, Koji Sakuraia, Satoshi Hirakob, Masahiro Wadaa AM. Protective effects of fish oil and pioglitazone on pancreatic tissue in obese KK mice with type 2 diabetes. Prostaglandins Leukot Essent Fat Acids. 2016;115:53-9.

11. Dhillon PK, Bowen L, Kinra S, Bharathi AV, Agrawal S, Prabhakaran D, et al. Legume consumption and its association with fasting glucose, insulin resistance and type 2 diabetes in the Indian Migration Study. Public Health Nutr. 2016;19(16):3017-26.

12. Lillioja $S$, Neal AL, Tapsell L JDJ. Whole Grains, Type 2 Diabetes, Coronary Heart Disease, and Hypertension: Links to the Aleurone preferred over Indigestible Fiber. Biofactors. 2013;39(3):242-58.

13. Fardet A. Minimally processed foods are more satiating and less hyperglycemic than ultra-processed foods: A preliminary study with 98 ready-to-eat foods. Food Funct. $2016 ; 7(5): 2338-46$.

14. Jakubowicz $D$, Wainstein J, Ahrén B, BarDayan Y, Landau Z, Rabinovitz HR, et al. High-energy breakfast with low-energy dinner decreases overall daily hyperglycaemia in type 2 diabetic patients: a randomised clinical trial. Diabetologia. 2015;58(5):912-9.

15. Lean ME TML. Sugar and Type 2 diabetes. Br Med Bull. 2016;120(1):43-53.

16. Engler PA, Ramsey SE SR. Alcohol use of diabetes patients: the need for assessment and intervention. Acta Diabetol. 2013;50(2):93-9.

17. Petrone $A B$, Gaziano JM, Link C. Consumption of Fried Foods and Risk of Heart Failure in the. $2016 ;(1): 1-7$.

18. Hollekim-Strand SM, Malmo V, Follestad T, Wisløff $U$, Ingul CB. Fast food increases postprandial cardiac workload in type 2 diabetes independent of pre-exercise: A pilot study. Nutr J. 2015;14(1):1-11.

19. Firouzi S, Barakatun-Nisak MY AK. Nutritional status, glycemic control and its associated risk factors among a sample of type 2 diabetic individuals, a pilot study. J Res Med Sci. 2015;20(1):40-6.

20. Kalra S. Diabesity. J Pak Med Assoc. 2013; 63(4):532-4. 